\title{
Crescimento de mudas de Poincianella pluviosa (DC.) L.P.Queiroz em diferentes substratos e lâminas de irrigação
}

\section{Growth seedlings of Poincianella pluviosa (DC.) L.P.Queiroz in different substrates and irrigation blades}

\author{
Victória Vieira Fantaus Pinto', Mateus Marques Bueno², Luiz Fernando de Sousa Antunes³, \\ Jorge Makhlouta Alonso ${ }^{4^{*}}$, e Gustavo Wyse Abaurre ${ }^{5}$
}

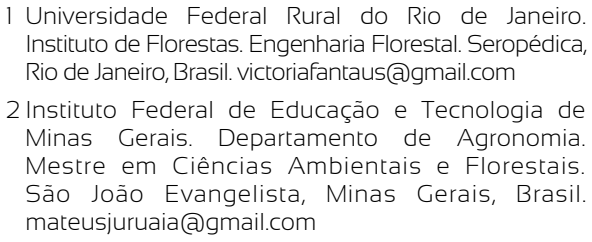
Instituto de Florestas. Engenharia Florestal. Seropédica, Rio de Janeiro, Brasil. victoriafantaus@gmail.com

2 Instituto Federal de Educação e Tecnologia de Minas Gerais. Departamento de Agronomia. Mestre em Ciências Ambientais e Florestais. São João Evangelista, Minas Gerais, Brasil. mateusjuruaia@gmail.com

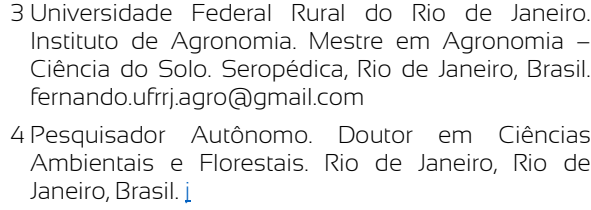

3 Universidade Federal Rural do Rio de Janeiro. Instituto de Agronomia. Mestre em Agronomia Ciência do Solo. Seropédica, Rio de Janeiro, Brasil fernando.ufrrj.agro(a)gmail.com

4 Pesquisador Autônomo. Doutor em Ciências Ambientais e Florestais. Rio de Janeiro, Rio de Janeiro, Brasil. j

\begin{abstract}
5 Universidade Federal Rural do Rio de Janeiro. Instituto de Florestas. Ciências Ambientais e Florestais. Seropédica, Rio de Janeiro, Brasil. gwabaurreagmail.com

* Autor de correspondencia. j_makh@hotmail.com
\end{abstract}

\section{RESUMO}

O Brasil tem hoje uma demanda por 21 milhões de hectares de restauração florestal, e como consequência, uma enorme demanda por mudas nativas. Desta forma, torna-se essencial o uso de técnicas que possam maximizar o uso dos substratos e da água de irrigação, visando a produção de mudas de alta qualidade. Diante deste cenário, o presente estudo teve como objetivo avaliar o crescimento de mudas de Poincianella pluviosa (DC.) L.P.Queiroz produzidas em quatro substratos elaborados a partir de diferentes resíduos (subsolo, lodo de esgoto, serragem compostada e moinha de carvão) e sob duas lâminas de irrigação $(0,03 \mathrm{~L} / \mathrm{h}$ e $0,05 \mathrm{~L} / \mathrm{h})$ por dia. O experimento foi conduzido em delineamento inteiramente casualizado com esquema fatorial $4 \times 2$ (quatro tipos de substratos e duas lâminas d'água), totalizando oito tratamentos. Os substratos foram avaliados quanto às suas características físicas e químicas. Foram mensuradas a altura, o diâmetro de colo e a massa seca da parte aérea e radicular das mudas após 105 dias de emergência. Os substratos S1- lodo de esgoto puro e S4- lodo de esgoto + serragem (1:1) apresentaram melhores características físicas e os substratos S1- lodo de esgoto puro e S3lodo de esgoto + moinha de carvão (1:1) exibiram melhor composição nutricional. Para crescimento em altura não foram observadas diferenças entre os substratos e lâminas de irrigação testados. Os substratos constituídos por $50 \%$ de lodo e $50 \%$ de moinha de carvão ou serragem e com a maior lâmina de irrigação resultaram em mudas com maior diâmetro, biomassa e qualidade, sendo os mais indicados à produção de $P$. pluviosa.

PALAVRAS CHAVE: biossólido, resíduos sólidos, restauração florestal, viveiros florestais.

\section{ABSTRACT}

Currently, Brazil has a demand for 21 million hectares of forest restoration, and consequently, a great demand for good quality native species seedlings. It is important to employ techniques that reduce the costs of productive inputs as substrates and the irrigation water. In this context, the paper aimed to evaluate the growth of Poincianella plwviosa (DC.) L.P.Queiroz seedlings produced in four substrates elaborated with different solid wastes (sewage sludge, composted sawdust, and charcoal powder) and under two irrigation depths $(0,03$ $\mathrm{L} / \mathrm{h}$ and $0,05 \mathrm{~L} / \mathrm{h}$ ). The experiment was conducted in completely randomized design with a $4 \times 2$ factorial arrangement (four substrates and two irrigation depths), eight treatments in total. The physical (porosity, density etc) and chemical (nutrients and organic matter contents) properties of substrates were evaluated. The seedlings shoot height, collar diameter, shoot, root, and total dry mass were measured. The substrates S1- pure sewage sludge and S2- sludge + sawdust (1:1) presented the better physical characteristics and substrates S1- pure sewage sludge and S3- sludge + charcoal powder (1:1) showed the better nutritional composition. For the growth in height there were no difference between the substrates and the irrigation depths. The substrates formulated with $50 \%$ of sludge and $50 \%$ of sawdust or charcoal powder, combined with the higher irrigation depth, resulted in seedlings with higher collar diameter, biomass, and quality, for which they are recommended to produce $P$. pluviosa.

KEYWORDS: biosolid, solid wastes, forest restoration, forest nurseries. 


\section{INTRODUÇÃO}

O processo de uso e ocupação dos solos no Brasil passou por muitas transformações ao longo de sua história, deixando como herança a degradação ambiental. Vastas áreas com vegetação nativa foram desmatadas para atender às demandas geradas pelo crescimento populacional, provocando a alteração dos biomas e de suas funções ecológicas. Com isso, existe a necessidade de promover a restauração florestal e de outros ecossistemas em diferentes regiões do país (Coutinho, Valcarcel, Rodrigues e Braga, 2019).

Devido a estes impactos, atualmente, o bioma Mata Atlântica apresenta grande parte de sua vegetação remanescente distribuída em pequenos fragmentos florestais isolados uns dos outros (Ribeiro, Metzger, Martensen, Ponzoni e Hirota, 2009), sendo que sua cobertura vegetal se limita em apenas $12,4 \%$ da original (SOS Mata Atlântica 2019). Só no estado do Rio de Janeiro, a área total de compromisso de restauração junto ao Instituto Estadual do Ambiente (INEA) é de aproximadamente 16000 ha, incluindo plantios não iniciados ou que foram iniciados, mas que se encontram em fase de manutenção (INEA, 2019).

A demanda por mudas de espécies florestais nativas, destinadas principalmente às atividades de restauração florestal, aumentou e com isso surge a necessidade de pesquisas que visem aprimorar os processos de produção. As principais metas são reduzir os custos de produção e aumentar a qualidade final das mudas, visando atender aos objetivos dos plantios (José, Davide e Oliveira, 2005). O sucesso dos plantios florestais está relacionado com a qualidade das mudas produzidas, a qual depende do tipo de recipiente, da qualidade das sementes, do substrato utilizado, dentre outros fatores (Caldeira, Peroni, Gomes, Delarmelina e Trazzi, 2012).

O substrato tem a função de dar sustentação para a planta, além de fornecer água, nutrientes e oxigênio durante o período de produção das mudas (Wendling e Gatto, 2012). A utilização de resíduos agrícolas, florestais, industriais ou urbanos, como matérias-primas na formulação de substratos, representa uma alternativa economicamente viável e ambientalmente adequada, tendo em vista que podem reduzir os custos do viveiro com a aquisição de substratos comerciais, além de promover o reaproveitamento de materiais que seriam descartados (Abreu et al. 2017b). Resíduos como lodo de esgoto, serragem e moinha de carvão já foram testados na produção de mudas de espécies florestais (Abreu et al. 2017a; Kratz, Nogueira, Wendling e Mellek, 2017; Caldeira, Rosa, Fenilli e Harbs, 2008), em geral, os estudos têm observado que esses materiais podem ser utilizados, desde que sejam observadas suas características e determinada a sua proporção adequada na composição dos substratos.

Outro fator que influencia o crescimento das mudas no viveiro é a irrigação, já que o mau planejamento pode resultar em perdas significativas para o viveiro. Por outro lado, o seu manejo correto é determinante para a qualidade das mudas, podendo reduzir o seu tempo de formação (Wendling e Gatto, 2012). A irrigação exerce influência direta na qualidade das mudas, a quantificação hídrica na fase de formação é muito importante, pois a falta ou o excesso prejudicam a produção (Morais, Susin, Vivian e Araújo, 2012; Silva et al., 2020).

A Poincianella pluviosa (DC.) L.P.Queiroz (sibipiruna), pertencente à família Fabaceae, subfamília Caesalpinioideae, é uma espécie nativa da Mata Atlântica, muito indicada para o paisagismo e arborização urbana, considerando suas características estéticas, floração e por ser resistente às pragas, doenças e poluição (Carvalho, 2008). Conhecida popularmente como sibipiruna, é uma árvore semidecídua, de médio a rápido crescimento, sendo por isso muito indicada para projetos de restauração florestal (Carvalho, 2008).

\section{OBJETIVOS}

O estudo teve como objetivo avaliar o crescimento de mudas de $P$. pluviosa em diferentes lâminas de irrigação e substratos compostos por lodo de esgoto e outros materiais. 


\section{MATERIAIS E MÉTODOS}

O experimento foi realizado durante outubro de 2018 até fevereiro de 2019 (126 dias), em um viveiro comercial, localizado aproximadamente nas coordenadas $22^{\circ} 46^{\prime} 36^{\prime \prime}$ $\mathrm{S}$ e $43^{\circ} 39^{\prime} 50^{\prime}$ ' O, e com altitude média de 20 metros. O clima da região é classificado como Aw (classificação Köppen), com altas temperaturas e chuvas no verão e clima seco com temperaturas amenas no inverno. As chuvas estão concentradas no período de novembro a março, com médias anuais para precipitação de $1213 \mathrm{~mm}$ e temperatura de $24,5^{\circ} \mathrm{C}$ (Carvalho, Silva, Folegatti, Costa e Cruz, 2006). As mudas foram produzidas sob telas agrícolas poliolefinas comerciais, que proporcionaram o sombreamento médio de $60 \%$ da luminosidade recebida.

As matérias-primas utilizadas para compor os substratos foram o lodo de esgoto, subsolo argiloso (barro), moinha de carvão e serragem compostada. O lodo de esgoto foi fornecido pela Cedae (Companhia Estadual de Águas e Esgoto) e era proveniente da Estação de Tratamento de Esgotos (ETE) da Ilha do Governador, localizada no Rio de Janeiro - RJ - Brasil. Essa ETE trata esgoto de origem domiciliar e possui sistema de tratamento secundário por lodos ativados. O lodo passa por adensamento, estabilização e depois por desidratação, ficando por mais de 90 dias em leitos de secagem a pleno sol, de onde sai com teor de umidade abaixo de $20 \%$. O subsolo argiloso foi adquirido localmente no comércio. A moinha de carvão foi fornecida pela empresa Pinga Fogo, esse resíduo é resultado do transporte e manuseio do carvão vegetal, onde ocorre fragmentação de parte do material em pedaços pequenos. A serragem foi fornecida pela empresa Bob Ambiental, sendo o resíduo proveniente de diferentes espécies madeireiras, bem como de chapas de aglomerados e MDF, tendo sido compostada por pelo menos 90 dias em ambiente protegido.

Estes materiais foram misturados nas proporções de volume (1:1), de modo a compor os substratos utilizados no experimento, sendo eles: S1 - lodo de esgoto puro; S2 lodo de esgoto + subsolo argiloso; S3 - lodo de esgoto + moinha de carvão e S4 - lodo de esgoto + serragem. Para garantir a homogeneização dos materiais, cada substrato formulado foi misturado em betoneira e peneirados em malha de $0,5 \mathrm{~cm} \times 0,5 \mathrm{~cm}$.

O manejo de irrigação foi realizado conforme procedimento já adotado no viveiro, que consiste no acionamento do sistema por 25 minutos três vezes ao dia, sendo realizada por meio de microaspersores invertidos. Para fins deste estudo utilizou-se o Setor A e Setor B de irrigação, que apresentam vazão média iguais a $0,03 \mathrm{~L} / \mathrm{h}$ e $0,05 \mathrm{~L} / \mathrm{h}$, respectivamente, obtidos através do teste de uniformidade de distribuição de água. O Coeficiente de Uniformidade (CUD) e Coeficiente de Uniformidade de Christiansen (CUC), calculados conforme metodologia proposta por Frizzone (2008), foi de 70 e 74 e de 81 e 88, para os setores A e B, respectivamente. Os tratamentos foram compostos pela combinação dos dois fatores, conforme ilustrado na tabela 1.

TABELA 1. Tratamentos compostos a partir de quatro substratos e dois setores de irrigação.

\begin{tabular}{ccc}
\hline Tratamento & Substrato & Setor de irrigação \\
\hline T1 & S1 & \\
T2 & S2 & A \\
T3 & S3 & \\
T4 & S4 & \\
T5 & S1 & B \\
T6 & S2 & \\
T7 & S3 & \\
T8 & S4 & \\
\hline
\end{tabular}

O delineamento experimental adotado foi o inteiramente casualizado em esquema fatorial $(4 \times 2)$. O primeiro fator correspondeu as quatro formulações de substratos e o segundo aos dois setores de irrigação, totalizando oito tratamentos. Para cada tratamento havia cinco repetições e cada unidade amostral foi composta por seis plantas, totalizando 240 mudas.

Foram coletados dados de pluviosidade da região através do site do Instituto Nacional de Meteorologia (Instituto Nacional de Meteorologia [Inmet], 2019) provenientes da Estação Meteorológica Automática de 
Seropédica. Os dados de pluviosidade diários foram somados a cada sete dias para formar a pluviosidade semanal, além disso foi somada a quantidade de dias de chuva em cada semana (Fig. 1).

As análises de nutrientes dos substratos foram realizadas no Laboratório Safrar - Análises Agrícolas, sendo quantificados os valores de $\mathrm{pH}$ e os teores de $\mathrm{P}, \mathrm{K}$, Ca, $\mathrm{Mg}$ e $\mathrm{Al}^{+3}, \mathrm{H}+\mathrm{Al}$, M.O., C.O., B, Cu, Fe, Mn e $\mathrm{Zn}$ disponíveis, de acordo com os procedimentos descritos por Raij, Andrade, Cantarella e Quaggio (2001).

A caracterização física constituiu na obtenção da curva de retenção da água contida em cada substrato, obtidas por meio do método de evaporação simplificado. Assim obteve-se a porosidade total, espaço de aeração, disponibilidade de água e densidade, conforme metodologia proposta por Fermino (2003).

As sementes de $P$. pluviosa foram coletadas a partir de arvores em bom estado fitofisiológico, localizadas no campus da Universidade Federal Rural do Rio de Janeiro Seropédica - RJ - Brasil. Aos 20 dias após a germinação, as plântulas foram repicadas para tubetes de $280 \mathrm{~cm}^{3}$, alocados em bandejas de 54 células na qual 100\% do espaço foi ocupado.

Quanto ao manejo de fertilização, cada muda recebeu cinco fertilizações de cobertura com $0,1 \mathrm{~g}$ de sulfato de amônia e 0,015 g de cloreto de potássio diluídos em $1 \mathrm{~mL}$ de água, aplicados na superfície do substrato com o auxílio de uma seringa. As adubações foram realizadas aos 33 dias, 47 dias, 60 dias, 74 dias e 88 dias após a repicagem das mudas.

As avaliações morfológicas das mudas ocorreram aos 35 dias, 70 dias e 105 dias após repicagem, com medição de diâmetro de colo (DC), com auxílio de paquímetro digital, e altura da parte aérea $(\mathrm{H})$, com uma régua graduada. Ao final do experimento, a parte aérea foi separada da raiz e esses materiais foram secos em estufa de circulação forçada a temperaturas entre $65^{\circ} \mathrm{C}$ e $70{ }^{\circ} \mathrm{C}$ por 72 horas. Os dados de massa seca de parte aérea (MSA) e massa seca de raiz (MSR) foram obtidos através da pesagem em balança de precisão com duas casas decimais. A massa seca total (MST) foi obtida a partir do somatório de MSR e MSA.

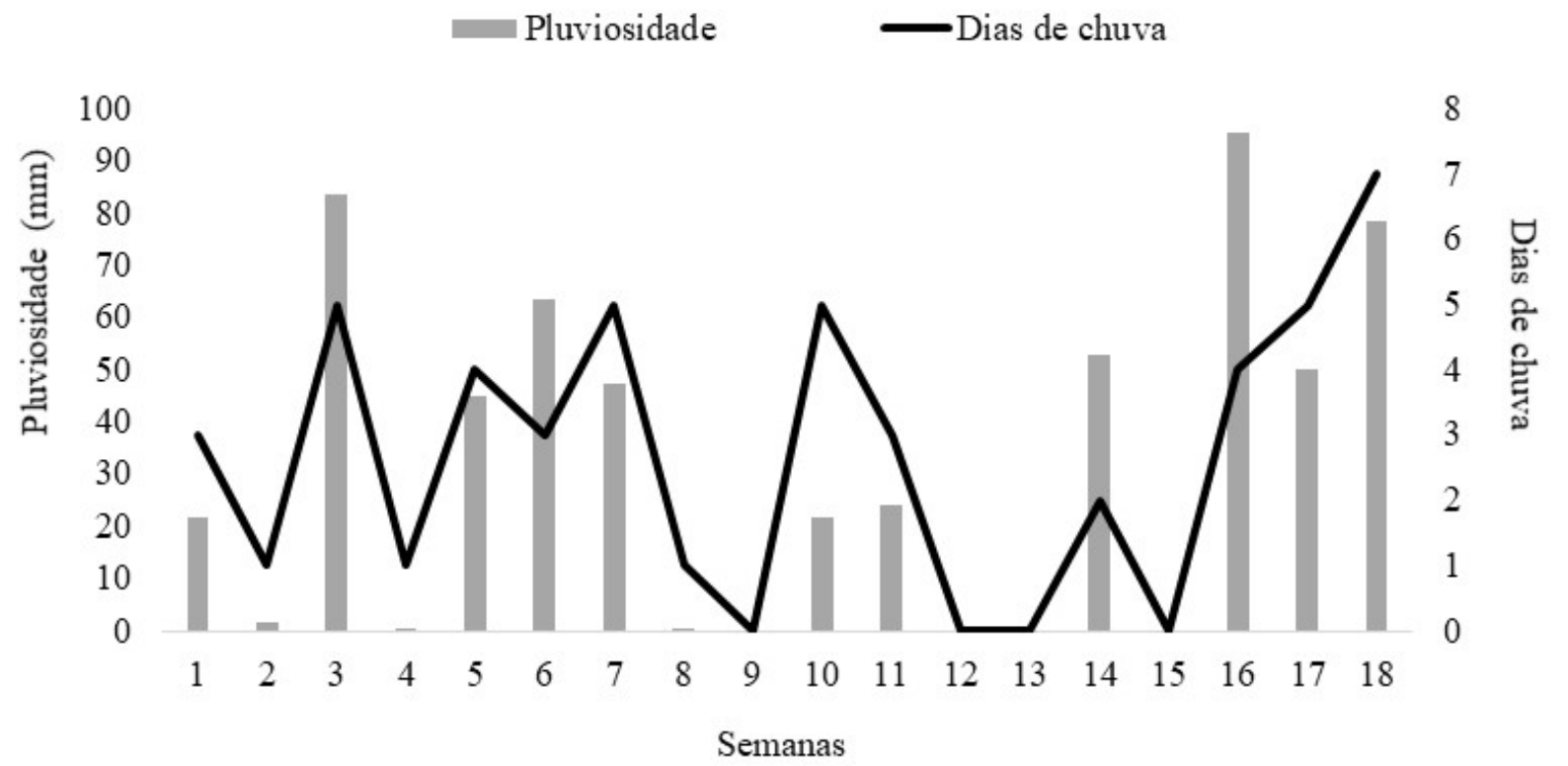

FIGURA 1. Pluviosidade (mm) e quantidade de dias de chuva ao longo das semanas em que foi conduzido o experimento, adaptado dos dados do Inmet (2019) 
A partir da mensuração dos dados acima foram calculados os parâmetros de qualidade: relação altura e diâmetro (HD); relação massa seca da parte aérea / massa seca de raiz (PAR) e o índice de qualidade de Dickson (IQD).

Os dados foram submetidos à análise de variância, após a qual a normalidade e a homogeneidade dos resíduos foram verificadas, respectivamente pelos testes de ShapiroWilk e de Bartlett. Posteriormente, os dados da avaliação das mudas foram submetidos ao teste de Tukey $(\mathrm{p}<0,05)$ com o auxílio do programa livre de estatística $\mathrm{R}$ ( $\mathrm{R}$ Core Team, 2020).

\section{RESULTADOS E DISCUSSÃO}

A densidade seca dos substratos situou-se entre $0,37 \mathrm{~g} / \mathrm{cm}^{3}$ e $1,04 \mathrm{~g} / \mathrm{cm}^{3}$, sendo o substrato S2 (lodo de esgoto + subsolo argiloso) aquele com maior valor (Tabela 2). Os valores de densidade seca recomendados por Kämpf (2005) variam de acordo com a altura dos recipientes, considerando os tubetes utilizados no presente estudo, com $19 \mathrm{~cm}$ de altura, valores entre $0,30 \mathrm{~g} / \mathrm{cm}^{3}$ e $0,50 \mathrm{~g} / \mathrm{cm}^{3}$ representariam substratos com densidade adequada. Nesse sentido, todos os substratos se encontram na faixa adequada, com exceção do S2, que apresentou valor bastante acima dos recomendados.

Substratos com densidades altas, como o S2, não são indicados para produção de mudas florestais em tubetes, podendo dificultar a drenagem da água de irrigação e limitar a quantidade de ar no substrato, causando asfixia radicular (Zapata, Guerrero e Polo, 2005). Além disso, a alta densidade resulta em um substrato mais pesado, o que dificulta a manipulação das mudas no viveiro e no transporte até a área de plantio (Cabreira et al. 2017). Como a produção em tubetes geralmente se dá em bandejas ou canteiros suspensos, é desejável que o substrato seja leve para facilitar o manuseio das mudas e não sobrecarregar a estrutura dos canteiros.

O substrato S1 (lodo de esgoto puro) foi o único que apresentou $\mathrm{pH}$ dentro da faixa ótima $(5,2-5,5)$, o substrato S2 poderia ser classificado com levemente ácido $(5,0-5,1)$ e os substratos S3 e S4 como levemente alcalinos $(5,6-5,8)$, de acordo com as recomendações de Kämpf (2005). Para espécies florestais, Gonçalves e Poggiani (1996) recomendam valores entre 5,5 e 6,5, com base nela os substratos S3 (lodo de esgoto + moinha de carvão) e S4 (lodo de esgoto + serragem) seriam os mais adequados.

Apenas os substratos S1 e S4 apresentaram porosidade total entre $75 \%$ e $85 \%$, considerada adequada para produção de mudas florestais de acordo com Gonçalves e Poggiani (1996). A porcentagem de macroporos se encontrou abaixo da recomendada pelos mesmos autores (35\% até $45 \%$ ) para todos os substratos e a de microporos (45\% até 55\%) acima. Nos trabalhos de Kratz, Wendling, Nogueira e Souza (2013) e Trigueiro e Guerrini (2014) à medida em que a proporção de lodo de esgoto em substratos aumentava, observou-se uma tendência de aumento da microporosidade e diminuição da macroporosidade, resultados similares aos observados no presente trabalho.

Em relação às características químicas, o substrato $\mathrm{S} 1$ apresentou valores maiores que os demais para $\mathrm{P}, \mathrm{Ca}, \mathrm{Cu}$, $\mathrm{Fe}, \mathrm{Zn}$ e $\mathrm{MO}$, resultado semelhante ao observado por Abreu et al. (2017b) em substrato formado por 100\% de lodo de esgoto, o que é justificado pelo fato deste material ser rico em matéria orgânica e nutrientes. O substrato S3 apresentou os maiores teores de $\mathrm{K}, \mathrm{Mg}$, B e Mn, semelhante ao observado por Souchie et al. (2011) em substrato com $50 \%$ de carvão moído para os nutrientes K, Mg e Mn. Em geral, o lodo de esgoto apresenta teores baixos de $\mathrm{K}$ em sua composição, considerando que esse elemento é altamente solúvel e permanece diluído na água durante o tratamento do esgoto (Berton e Nogueira, 2010). A análise química do substrato $\mathrm{S} 3$ demonstra que a moinha de carvão se mostrou um material adequado para a finalidade de adicionar $\mathrm{K} \mathrm{em}$ substratos formulados com lodo de esgoto.

Em relação ao alumínio trocável $(\mathrm{H}+\mathrm{Al})$, apenas os substratos S1 e S2 apresentaram teores consideráveis. Porém, quando o pH do solo está entre 5,2 e 5,3 (Tabela 2) o alumínio trocável se encontra insolubilizado, ou seja, quase incapaz de causar danos às raízes (Sobral, Barreto, Silva e Anjos, 2015), não sendo esse parâmetro um fator de restrição para o desenvolvimento das mudas em nenhum dos substratos. 
TABELA 2. Valores médios do pH e das características químicas e físicas dos substratos S1 (lodo de esgoto puro), S2 (lodo + terra de subsolo), S3 (lodo + moinha de carvão) e S4 (lodo + serragem).

\begin{tabular}{|c|c|c|c|c|c|}
\hline & \multirow{2}{*}{ Parâmetros } & \multicolumn{4}{|c|}{ Substratos } \\
\hline & & $S 1$ & $S 2$ & S3 & 54 \\
\hline \multirow{14}{*}{ Químicos } & $\mathrm{pH}\left(\mathrm{H}_{2} \mathrm{O}\right)$ & 5,3 & 5,0 & 5,6 & 5,8 \\
\hline & $P(\mathrm{mg} / \mathrm{L})$ & 141,1 & 55,3 & 112,8 & 78,6 \\
\hline & $\mathrm{K}(\mathrm{mg} / \mathrm{L})$ & 39 & 43 & 431 & 72 \\
\hline & $\mathrm{Ca}\left(\mathrm{mmol}_{\mathrm{c}} \mathrm{dm}^{3}\right)$ & 56 & 27 & 49 & 44 \\
\hline & $\mathrm{Mg}\left(\mathrm{mmol}_{\mathrm{c}} \mathrm{dm}^{3}\right)$ & 7 & 8 & 32 & 15 \\
\hline & $\mathrm{Al}\left(\mathrm{mmol}_{\mathrm{c}} \mathrm{dm}^{3}\right)$ & 2 & 2 & 1 & 0 \\
\hline & $\mathrm{H}+\mathrm{Al}\left(\mathrm{mmol}_{\mathrm{c}} \mathrm{dm}^{3}\right)$ & 47 & 50 & 29 & 22 \\
\hline & M.O. (g/kg) & 63 & 29 & 57 & 51 \\
\hline & C.O. (g/kg) & 36,5 & 17,1 & 33,2 & 29,4 \\
\hline & $\mathrm{B}(\mathrm{mg} / \mathrm{L})$ & 1,86 & 1,06 & 2,66 & 0,89 \\
\hline & $\mathrm{Cu}(\mathrm{mg} / \mathrm{L})$ & 14,2 & 4,7 & 6,2 & 5,8 \\
\hline & $\mathrm{Fe}(\mathrm{mg} / \mathrm{L})$ & 65 & 38 & 29 & 23 \\
\hline & $\mathrm{Mn}(\mathrm{mg} / \mathrm{L})$ & 17,8 & 7,4 & 20,8 & 6,1 \\
\hline & $\mathrm{Zn}(\mathrm{mg} / \mathrm{L})$ & 10,8 & 5,0 & 7,1 & 5,1 \\
\hline \multirow{7}{*}{ Físicos } & Densidade úmida $\left(\mathrm{g} / \mathrm{cm}^{3}\right)$ & 0,81 & 1,42 & 0,64 & 0,64 \\
\hline & Densidade seca $\left(\mathrm{g} / \mathrm{cm}^{3}\right)$ & 0,49 & 1,04 & 0,41 & 0,37 \\
\hline & Densidade de partícula $\left(\mathrm{g} / \mathrm{cm}^{3}\right)$ & 2,4 & 2,7 & 1,9 & 1,7 \\
\hline & Porosidade total (\%) & 79,21 & 68,27 & 65,54 & 81,61 \\
\hline & Macroporosidade (\%) & 10,37 & 16,45 & 9,36 & 11,47 \\
\hline & Microporosidade (\%) & 68,84 & 51,82 & 56,18 & 70,14 \\
\hline & $C R A_{10} \mathrm{~cm}$ & 117,73 & 156,72 & 105,88 & 103,83 \\
\hline
\end{tabular}

Onde: M.O. - matéria orgânica; C.O. - carbono orgânico; CRA - capacidade de retenção de água.

Considerando os parâmetros avaliados, os substratos $\mathrm{S} 1$ e S4, apresentaram as melhores características físicas, considerando que seus valores de densidade e porosidade estavam dentro dos recomendados pela literatura. Para as características químicas, os dois melhores substratos foram o S1 e S3, os quais apresentaram maiores teores de nutrientes e de matéria orgânica, sendo que o S1 continha principalmente mais $\mathrm{P}$ e micronutrientes, enquanto o S3 continha mais $\mathrm{Ke} \mathrm{Mg}$.
A análise de variância demonstrou que para todos as variáveis avaliadas não houve interação significativa entre os fatores substrato e irrigação. Para os fatores isolados, não foram observadas diferenças entre os tratamentos para as variáveis altura, relação altura e diâmetro (HD) e relação parte aérea e raiz (PAR) entre substratos, nem para altura e HD entre os setores de irrigação (Tabela 3). Para as demais variáveis foram verificadas diferenças entre tratamentos de ambos fatores. 
Para a altura da parte aérea, as mudas de $P$. pluviosa apresentaram valores médios entre $24,82 \mathrm{~cm}$ e $29,11 \mathrm{~cm}$ para substratos e 25,91 cm e 28,40 cm para irrigação (Tabela 3). Além de não apresentar diferenças pela estatística, todos os tratamentos se adequaram ao intervalo recomendado por Gonçalves, Petri, Caldeira, Dalmaso e Silva (2014), entre $20 \mathrm{~cm}$ e $35 \mathrm{~cm}$, para mudas de espécies florestais de boa qualidade. Quando atingem de $25 \mathrm{~cm}$ a $30 \mathrm{~cm}$ de altura, Moraes, Assumpção, Pereira e Luchiari (2013) mencionam que mudas de espécies nativas da Mata Atlântica estão prontas para plantio de restauração florestal. Portanto, considerando esse parâmetro, todas os tratamentos produziram mudas de qualidade adequada e prontas para o plantio.

Verificou-se valores médios de diâmetro do colo superiores nos substratos S3 e S4 em relação ao substrato S1. Para o substrato S2 não se observou diferença em relação aos demais tratamentos (Tabela 3). Os substratos S3 e S4 apresentaram os maiores teores de potássio, nutriente escasso no lodo de esgoto, mas presente na serragem e principalmente na moinha de carvão. Esta característica pode ter contribuído para o maior crescimento em diâmetro nesses substratos, visto que o teor de potássio pode influenciar o crescimento dessa variável (Gomes e Paiva, 2006), conforme observado para Plathymenia foliososa por Duarte et al. (2015). Para espécies nativas, Gonçalves et al. (2000) sugerem que mudas de qualidade devem apresentar diâmetro entre $5 \mathrm{~mm}$ e $10 \mathrm{~mm}$. Dessa forma, ao contrário do que foi observado para altura, nenhum dos tratamentos avaliados teria proporcionado mudas com qualidade adequada, seja para substrato ou para irrigação.

O mesmo padrão observado para diâmetro ocorreu para a variável massa seca total (MTS), onde os substratos S3 e S4 foram superiores ao S1, não havendo diferença entre o S2 e os demais tratamentos (Tabela 3). Quanto a massa seca da parte aérea (MAS), o substrato S4 obteve média superior aos S1 e S2, enquanto o S3 não diferiu dos demais substratos. A massa seca de raiz (MRS) foi maior no substrato S3 em relação aos S1 e S2 e semelhante ao substrato $\mathrm{S} 4$, que por sua vez apresentou média superior ao S1 e semelhante ao S2. De modo geral, a maior produção de biomassa foi observada nas mudas dos substratos S3 e S4. Segundo Gomes e Paiva (2006), a MAS é um indicador da rusticidade das mudas, enquanto a MRS é um dos melhores e mais importantes parâmetros para estimar a sobrevivência e crescimento inicial das mudas após o plantio, dessa forma, as mudas dos substratos S3 e S4 seriam as mais rústicas e adequadas para plantio aos 105 dias após a repicagem.

TABELA 3. Médias de altura, diâmetro, massa seca da parte aérea (MAS), radicular (MRS) e total (MTS), relação altura e diâmetro (HD), parte aérea e raiz (PAR) e índice de qualidade de Dickson (IQD) das mudas de Poincianella pluviosa aos 105 dias após a repicagem em diferentes substratos e lâminas de irrigação.

\begin{tabular}{ccccc|cc}
\hline & \multicolumn{4}{c|}{ Substratos } & \multicolumn{2}{c}{ Irrigação } \\
& S1 & S2 & S3 & S4 & A & B \\
\hline Altura (cm) & $26.26 \mathrm{~ns}$ & $24.82 \mathrm{~ns}$ & $28.42 \mathrm{~ns}$ & $29.11 \mathrm{~ns}$ & $25.91 \mathrm{~ns}$ & $\mathrm{~ns}$ \\
Diâmetro (mm) & $3.38 \mathrm{~b}$ & $3.54 \mathrm{ab}$ & $3.90 \mathrm{a}$ & $3.88 \mathrm{a}$ & $3.42 \mathrm{~b}$ & $3.93 \mathrm{a}$ \\
MAS (g) & $2.40 \mathrm{~b}$ & $2.53 \mathrm{~b}$ & $3.39 \mathrm{ab}$ & $3.66 \mathrm{a}$ & $2.64 \mathrm{~b}$ & $3.35 \mathrm{a}$ \\
MRS (g) & $0.66 \mathrm{c}$ & $0.86 \mathrm{bc}$ & $1.21 \mathrm{a}$ & $1.07 \mathrm{ab}$ & $0.76 \mathrm{~b}$ & $1.14 \mathrm{a}$ \\
MTS (g) & $3.07 \mathrm{~b}$ & $3.39 \mathrm{ab}$ & $4.60 \mathrm{a}$ & $4.73 \mathrm{a}$ & $3.40 \mathrm{~b}$ & $4.49 \mathrm{a}$ \\
HD & $5.99 \mathrm{~ns}$ & $5.65 \mathrm{~ns}$ & $5.66 \mathrm{~ns}$ & $5.58 \mathrm{~ns}$ & $5.60 \mathrm{~ns}$ & $5.83 \mathrm{~ns}$ \\
PAR & $3.36 \mathrm{~ns}$ & $3.00 \mathrm{~ns}$ & $3.08 \mathrm{~ns}$ & $3.49 \mathrm{~ns}$ & $3.48 \mathrm{a}$ & $2.98 \mathrm{~b}$ \\
IQD & $0.32 \mathrm{c}$ & $0.40 \mathrm{bc}$ & $0.57 \mathrm{a}$ & $0.53 \mathrm{ab}$ & $0.38 \mathrm{~b}$ & $0.53 \mathrm{a}$ \\
\hline
\end{tabular}

Letras iguais na mesma linha não diferem estatisticamente entre si pelo teste de Tukey a 5\% de probabilidade. Para substratos S1 (lodo de esgoto puro), S2 (lodo + subsolo), S3 (lodo + moinha de carvão); 44 (lodo + serragem); e para irrigação A equivale a lâmina de 0,03 L/h e B a lâmina de 0,05 L/h 
Quanto à irrigação, contata-se que o crescimento em diâmetro foi maior no setor $\mathrm{B}$, onde a lâmina de irrigação era maior (Tabela 3). O que também foi observado para as variáveis de biomassa (MAS, MRS e MST). Considerando que a deficiência hídrica provoca estresse nas plantas, diminui a absorção de nutrientes e por consequência o crescimento e acúmulo de biomassa (Morais et al. 2012) é possível afirmar que a quantidade de água ministrada no setor A foi restritiva para o crescimento das mudas. O maior crescimento em diâmetro e acúmulo de biomassa em lâminas de irrigação maiores é um padrão observado por outros autores para mudas de espécies leguminosas arbóreas, como Sabonaro e Galbiatti (2011) para Schizolobium parabyba e Silva, Tagliaferre, Paula, Lemos e Rocha, 2019 para Anadenanthera colubrina, bem como para outras espécies florestais (Scalon, Mussury, Euzébio, Kodama e Kissmann, 2011; Silva et al., 2019). No entanto, conforme recomendado por Morais et al. (2012) e Gomes e Paiva (2006) a água deve ser usada de forma racional, visando a economia desse recurso por vezes escasso, bem como evitar a lixiviação de nutrientes do substrato com o excesso de água da irrigação.

Dentre os parâmetros de qualidade das mudas de $P$. pluviosa apenas o índice de qualidade de Dickson (IQD) apresentou médias diferentes entre os substratos avaliados (Tabela 3). O substrato S3 apresentou IQD maior que S1 e S2, enquanto para o S4 o IQD foi maior que o de S1 e similar ao de S2 e S3. Quanto aos setores de irrigação, maior média de IQD foi observada para a maior lâmina d'água e o inverso foi observado para a PAR. A relação HD foi semelhante em todos os tratamentos avaliados, tanto para substrato como para irrigação, com valores médios entre 5,5 e 6,0. Segundo Araujo et al. (2018), valores de HD entre 2 e 8 podem ser considerados adequados para mudas de espécies nativas. Dessa forma, tendo HD como parâmetro, todos os tratamentos avaliados no presente estudo produziram mudas de qualidade adequada.

A PAR está relacionada com o balanço hídrico das mudas, valores altos podem indicar mudas mais suscetíveis a estresse hídrico, considerando a desigualdade de proporção entre a sua superfície de transpiração (parte aérea) e seu potencial de absorver água (raiz) (Grossnickle, 2012). Araujo et al. (2018) mencionam que os valores de PAR para espécies nativas devem variar de 1 a 3 , o que no presente estudo ocorreu apenas para o substrato S2 e o setor B de irrigação. Os demais tratamentos apresentaram valores um pouco maiores que 3 , entre 3,08 e 3,48, sendo próximos do adequado.

Em relação ao IQD, Gomes e Paiva (2006) mencionam que quanto maior o seu valor, maior a qualidade das mudas produzidas, sendo que a partir de valores de 0,20, mudas de algumas espécies utilizadas na silvicultura comercial podem ser consideradas de qualidade adequada. Tendo como referência o valor de 0,20 todos os tratamentos avaliados no presente estudo produziram mudas adequadas para plantio. No entanto, esse parâmetro deve ser utilizado com cuidado já que os dados existentes são insuficientes e não existem valores de referência para espécies nativas da Mata Atlântica (Alonso et al., 2018).

Conforme mencionam Gomes e Paiva (2006), Araujo et al. (2018), Alonso et al. (2018), dentre outros autores, nenhum parâmetro deve ser avaliado isoladamente como indicativo de qualidade de mudas florestais. Considerando em conjunto os parâmetros avaliados neste estudo, todos os tratamentos apresentaram valores médios de altura, HD e IQD dentro dos adequados para mudas florestais. Apesar disto, como maior crescimento e qualidade foram observados nos substratos S3 e S4 e no setor B de irrigação, esses seriam os tratamentos mais indicados para a produção de mudas de P. pluviosa.

Em outro estudo com produção de mudas da espécie P. pluviosa, Pinto, Souza, Novaes, Paula e Aguiar Junior (2017) testaram diferentes recipientes, observando melhores resultados para o tubete de $288 \mathrm{~cm}^{3}$ (o mesmo utilizado no presente estudo), com médias de $15,91 \mathrm{~cm}$ para altura, 3,67 mm para diâmetro, 4,36 para $\mathrm{HD}, 1,82 \mathrm{~g}$ para MAS e $0,99 \mathrm{~g}$ para MRS aos 120 dias após a semeadura. Os valores observados nos melhores tratamentos do presente estudo (substratos S3 e S4 e setor B de irrigação) foram superiores aos de Pinto et al. (2017), com exceção do diâmetro no setor $\mathrm{B}$, demonstrando a qualidade das mudas produzidas. Por outro lado, os resultados do presente 
estudo foram inferiores aos observados por Oliveira et al. (2017) para mudas de P. pluviosa em diferentes substratos, aos 120 dias após o transplante. Os autores observaram melhores resultados para substrato contendo $75 \%$ de solo, $15 \%$ de areia e $10 \%$ de composto orgânico, com médias de $90,80 \mathrm{~cm}$ para altura, $11,72 \mathrm{~mm}$ de diâmetro, $18,57 \mathrm{~g}$ de MRS, 24,21 g de MAS, 42,78 g de MTS e 3,91 de IQD. Uma das justificativas para as diferenças observadas são os recipientes utilizados em cada estudo, já que Oliveira et al. (2017) utilizaram vasos plásticos com volume de 3 litros e o presente estudo tubetes de $288 \mathrm{~cm}^{3}$. Conforme observado por Leles, Lisboa, Oliveira Neto, Grugiki e Ferreira (2006), mudas florestais com tempo de produção similares tendem a apresentar maiores parâmetros morfológicos em recipientes maiores, tendo em vista a menor restrição radicular, o maior espaço e quantidade de substrato disponível para captação de água e nutrientes.

Observou-se que o substrato S1, com 100\% de lodo de esgoto, embora tenha apresentado teores de nutrientes superiores aos demais substratos e propriedades físicas adequadas, demonstrou menor crescimento em diâmetro e biomassa, além de mudas de $P$. plwiosa com qualidade inferior às produzidas nos substratos S3 e S4. Produzindo mudas da mesma espécie que o presente trabalho, Oliveira et al. (2017) observaram os melhores resultados de crescimento e qualidade para substratos formulados com a combinação de diferentes materiais (proporções variáveis de solo, composto orgânico e areia). Os autores verificaram ainda que as mudas produzidas em substrato com 100\% de composto orgânico apresentaram resultados dentre os piores. Os resultados de Oliveira et al. (2017) possuem certa similaridade com os do presente estudo, sugerindo que para a produção de mudas de $P$. pluviosa não é recomendado utilizar apenas composto orgânico como substrato, mas sim a mistura deste com outros materiais.

Estudando substratos com diferentes proporções de lodo de esgoto para a produção de mudas de Schinus terebinthifolia e Handroanthus heptaphyllus, Abreu, Leles, Melo, Oliveira e Ferreira (2017a) observaram maior crescimento de S. terebinthifolia em substratos com mais de 50\% de lodo, enquanto para $H$. heptaphyllus o maior crescimento foi observado em substrato com 50\% de lodo. Os autores atribuem essas diferenças às características fisiológicas e, por consequência, ao grupo ecológico das espécies. $\mathrm{O}$ substrato com 100\% de lodo apresentava maior capacidade de retenção de água e nutrientes, característica que foi bem aproveitada pela espécie rústica e de rápido crescimento $S$. terebinthifolia. Enquanto H. heptaphyllus, espécie secundária que não demanda altas concentrações de nutrientes e é adaptada a solos secos e bem drenados, não se beneficiou dessas características. Acredita-se que efeito semelhante possa ter ocorrido no presente estudo, tendo em vista que a $P$. pluviosa também é uma espécie secundária, de crescimento moderado a lento, adaptada a solos bem drenados e de baixa a média fertilidade (Carvalho, 2008). Tais resultados sugerem que, para espécies secundárias, não seja recomendado utilizar substratos com proporções maiores que $50 \%$ de lodo de esgoto.

A resposta observada para $P$. pluviosa foi semelhante a outros trabalhos que utilizaram lodo de esgoto como componente de substrato para produção de mudas de diferentes espécies de leguminosas arbóreas, como Cabreira et al. (2017) e Silva et al. (2020) para Peltophorum dubium, Caldeira et al. (2012) para Ateleia glazioveana e Faria, Caldeira, Delarmelina e Gonçalves (2013) para Mimosa setosa. Em geral, os autores relatam que o acréscimo desse material em substratos favorece o crescimento e a qualidade das mudas, tendo em vista seus altos teores de nutrientes e matéria orgânica. No entanto, Kratz, Nogueira, Wendling e Souza (2015), trabalhando com produção de mudas de Mimosa scabrella, observaram que substratos com proporções acima de $10 \%$ de lodo não proporcionaram mudas aptas para plantio em campo. Resultado semelhante ao de Siqueira et al. (2019) que não recomendaram proporções maiores que $20 \%$ de lodo em substratos para produção de mudas de Plathymenia reticulta. Conforme verificado por Abreu et al. (2017b), lodos de diferentes ETEs, com diferentes bacias de esgotamento, sistemas de tratamento, dentre outros fatores, podem apresentar características químicas e físicas diferentes, sendo mais ou menos adequados para aplicação na produção de mudas. A viabilidade de utilização do lodo de esgoto e a sua proporção na composição de substratos 
deve ser avaliada de acordo as características das espécies que se deseja produzir e de cada lote deste material (Alonso et al., 2018).

\section{CONCLUSÕES}

Os substratos S1 (lodo de esgoto puro) e S4 (lodo de esgoto + serragem), apresentaram melhores características físicas para a produção de mudas florestais. Quanto às características químicas, os substratos S1 e S3 (lodo de esgoto + moinha de carvão) obtiveram melhores resultados.

Os substratos S3 e S4 resultaram em mudas de melhor qualidade, com maior diâmetro e biomassa, sendo os mais indicados para a produção de mudas da espécie Poincianella pluviosa (DC.) L.P.Queiroz. Quanto à irrigação, os melhores resultados foram observados para as mudas produzidas sob a maior lâmina d'água (setor B).

\section{AGRADECIMENTOS}

À GWA Empreendimentos Florestais LTDA, pelo apoio técnico e financeiro. À CEDAE, Pinga Fogo e Bob Ambiental, pelo fornecimento dos materiais utilizados na composição dos substratos estudados.

\section{REFERÊNCIAS}

Abreu, A. H. M., Leles, P. S. S., Melo, L. A., Oliveira, R. R, \& Ferreira, D. H. A. A. (2017a). Caracterização e potencial de substratos formulados com biossólido na produção de mudas de Schinus terebinthifolius e Handroanthus heptaphyllus (Vell.) Mattos. Ciência Florestal, 27(4), 1179-1190. doi: 10.5902/1980509830300

Abreu, A. H. M., Marzola, L. B., Melo, L. A., Leles, P. S. S., Abel, E. L. S., \& Alonso, J. M. (2017b). Urban solid waste in the production of Lafoensia pacari seedlings. Revista Brasileira de Engenharia Agricola e Ambiental, 21(2), 83-87. doi: 10.1590/18071929/agriambi.v21n2p83-87

Alonso, J. M., Abreu, A. H. M., Melo, L. A., Leles, P. S. S., \& Cabreira, G. V. (2018). Biosolids as substrate for the production of Ceiba speciosa seedlings. Cerne, 24(4), 420-429. doi: 10.1590/01047760201824042568

Araujo, M. M., Navroski, M. C., Schorn, L. A., Tabaldi, L. A., Rorato, D. G., Turchetto, F., Zavistanovicz, T. C., Berghetti, A. L. P., Aimi, S. C., Tonetto, T. S., Gasparin, E., Kelling, M. B., Ávila, A. L., Dutra, A. F., Mezzomo, J. C., Gomes, D. R., Griebeler, A. M.,
Silva, M.R., Barbosa, F.M. \& Lima, M.S. (2018). Caracterização e análise de atributos morfológicos e fisiológicos indicadores da qualidade de mudas em viveiro florestal. Em M. M. Araujo, M. C. Navroski, \& L. A. Schorn (Eds.), Produção de sementes e mudas, um enfoque à silvicultura (pp. 345-366). Santa Maria (RS), Brasil: Editora UFSM.

Berton, R. S., \& Nogueira T. A. R. (2010). Uso de lodo de esgoto na agricultura. Em A. R. Coscione, T. A. R. Nogueira, \& A. M. M. Pires. Uso agrícola de lodo de esgoto: avaliação após a resolução $n^{\circ} 375$ do CONAMA (pp. 31-50). Botucatu, Brasil: Fundação de Estudos e Pesquisas Agrícolas e Florestais.

Cabreira, G. V., Leles, P. S. S., Alonso, J. M., Abreu, A. H. M., Lopes, N. F., \& Santos, G. R. (2017). Biossólido como componente de substrato para produção de mudas florestais. Revista Floresta, 47(2), 165-176. doi: 10.5380/rf.v47i2.44291

Caldeira, M. V. W., Rosa, G. N., Fenilli, T. A. B., \& Harbs, R. M. P. (2008). Composto orgânico na produção de mudas de aroeiravermelha. Scientia Agraria, 9(1), 27-33. doi: 10.5380/rsa.v9i1.9898

Caldeira, M. V. W., Peroni, L., Gomes, D. R., Delarmelina, W. M., \& Trazzi, P. A. (2012). Diferentes proporções de biossólido na composição de substratos para a produção de mudas de timbó (Ateleia glazioveana Baill). Scientia Forestalis, 40(93), 15-22.

Carvalho, D. F., Silva, L. D. B., Folegatti, M. V., Costa, J.R., \& Cruz, F. A. (2006). Avaliação da evapotranspiração de referência na região de Seropédica-RJ, utilizando lisímetro de pesagem. Revista Brasileira de Agrometeorologia, 14(2), 108-116.

Carvalho, P. E. R. (2008). Espécies Arbóreas Brasileiras Volume 3 (1ª ed.) Brasília, Brasil: Empresa Brasileira de Pesquisa Agropecuária (Embrapa).

Coutinho, P. R. O. S., Valcarcel, R., Rodrigues, P. J. F. P., \& Braga, J. M. A. (2019). Restauração passiva em pastagens abandonadas a partir de núcleos de vegetação na Mata Atlântica, Brasil. Ciência Florestal, 29(3), 1307-1323. doi: 10.5902/1980509827844

Duarte, M. L., Paiva, H. N., Alves, M. O., Freitas, A. F., Maia, F. F., \& Goulart, L. M. L. (2015). Crescimento e qualidade de mudas de vinhático (Platymenia foliolosa Benth.) em resposta à adubação com potássio e enxofre. Ciência Florestal, 25(1), 221-229. doi: $10.5902 / 1980509817480$

Faria, J. C. T., Caldeira, M. V. W., Delarmelina, W. M., \& Gonçalves, E. O. (2013). Uso de resíduos orgânicos no crescimento de mudas de Mimosa setosa. Pesquisa Florestal Brasileira, 33(76), 409-418. doi: 10.4336/2013.pfb.33.76.501 
Fermino, M. H. (2003). Métodos de análise para caracterização física de substratos para plantas. Tese, Universidade Federal do Rio Grande do Sul, Porto Alegre (RS), Brasil.

Frizzone, J. A. (2008). Uniformidade e Eficiência. ESALQ/USP, Piracicaba (SP), Brasil. Disponível em: http://www.leb.esalq.usp.br/leb/disciplinas/Frizzone/LEB_15 $71 /$

Gomes, J. M., \& Paiva, H. N. (2006). Viveiros florestais (propagação sexuada) (1 ${ }^{\mathrm{a}}$ ed.). Viçosa (MG), Brasil: Editora UFV.

Gonçalves, L. M., \& Poggiani, F. (1996). Substratos para produção de mudas florestais. Em Congresso Latino-Americano de Ciência do Solo, 13. Resumos..., Piracicaba (SP), Brasil: Sociedade LatinoAmericana de Ciência do Solo, CD-ROM.

Gonçalves, J. L. M., Santarelli, E. D., Neto, S. P. M., \& Manara, M. P. (2000). Produção de mudas de espécies nativas: substrato, nutrição, sombreamento e fertilização. Em J. L. M. Gonçalves, \& V. Benedetti (Eds.), Nutrição e fertilização florestal (pp. 309-350). Piracicaba (SP), Brasil: IPEF.

Gonçalves, O. E., Petri, G. M., Caldeira, M. V. W., Dalmaso, T. T., \& Silva, A. G. (2014) Crescimento de mudas de Ateleia glazioviana em substratos contendo diferentes materiais orgânicos. Floresta $e$ Ambiente, 21(3), 339-348. doi: 10.1590/2179-8087.029213

Grossnickle, S. C. (2012). Why seedlings survive: influence of plants attributes. New Forests, 43(1), 711-738. doi: 10.1007/s11056-0129336-6

Instituto Estadual do Ambiente [Inea]. (2019). Observatório Florestal Fluminense. Disponível em: https://www.restauracaoflorestalrj. org/observatorio

Instituto Nacional de Meteorologia [Inmet]. (2019). Dados Meteorológicos. Disponível em: http://www.inmet.gov.br/portal/

José, A. C., Davide, A. C., \& Oliveira, S. L. (2005). Produção de mudas de aroeira (Schinus terebinthifolius Raddi.) para recuperação de áreas degradadas pela mineração de bauxita. Revista Cerne, 11(2), 187196.

Kämpf, A. N. (2005). Produção comercial de plantas ornamentais ( $2^{\mathrm{a}}$ ed.). Guaíba (RS), Brasil: Agrolivros.

Kratz, D., Wendling, I., Nogueira, A.C., \& Souza, P. V. (2013). Propriedades físicas e químicas de substratos renováveis. Revista Árvore, 37(6), 1103-1113. doi: 10.5380/rf.v45i2.31249

Kratz, D., Nogueira, A. C., Wendling, I., \& Souza, P. V. D. (2015). Substratos renováveis para produção de mudas de Mimosa scabrella. Revista Floresta, 45(2), 393-408. doi: $10.5380 /$ rf.v45i2.31249
Kratz, D., Nogueira, A. C., Wendling, I., \& Mellek, J. E. (2017). Physicchemical properties and substrate formulation for Eucalyptus seedlings production. Scientia Forestalis, 45(113), 63-76. doi: 10.18671 /scifor.v45n113.06

Leles, P. S. S., Lisboa, A. C., Oliveira Neto, S. N., Grugiki, M. A., \& Ferreira, M. A. (2006). Qualidade de mudas de quatro espécies florestais produzidas em diferentes tubetes. Floresta e Ambiente, 13(1), 69-78.

Moraes, L. F. D., Assumpção, J. M., Pereira, T. S., \& Luchiari, C. (2013). Manual técnico para a restauração de áreas degradadas no Estado do Rio de Janeiro (1 ${ }^{\mathrm{a}}$ ed.). Rio de Janeiro (RJ), Brasil: Instituto de Pesquisas Jardim Botânico do Rio de Janeiro.

Morais, W. W. C., Susin, F., Vivian, M. A., \& Araújo, M. M. (2012). Influência da irrigação no crescimento de mudas de Schinus terebinthifolius. Pesquisa Florestal Brasileira, 32(69), 23-28. doi: 10.4336/2012.pfb.32.69.23

Oliveira, D. M., Caron, B. O., Elli, E. F., Monteiro, G. C., Schwerz, F., Basso, C. J., \& Manfron, P.A. (2017). Production and quality of Caesalpinia pluviosa seedlings in different substrates. Cientifica, 45(1), 1-8. doi: 10.15361/1984-5529.2017v45n1p1-8

Pinto, L., Souza, D., Novaes, A., Paula, R., \& Júnior, A. (2017). Qualidade de mudas de Caesalpinia peltophoroides Benth. produzidas em diferentes recipientes. Enciclopédia Biosfera, 14(25), 1095-1104. doi: 10.18677/EnciBio_2017A88

Raij, B., Andrade, J. C., Cantarella, H., \& Quaggio, J. A. (2001). Análise química para avaliação da fertilidade de solos tropicais. São Paulo (SP), Brasil: Editora IAC.

R Core Team (2020). R: A language and environment for statistical computing. $\mathrm{R}$ Foundation for Statistical Computing, Vienna, Austria. https://www.R-project.org/

Ribeiro, M. C., Metzger, J. P., Martensen, A. C., Ponzoni, F. J., \& Hirota, M. M. (2009). The Brazilian Atlantic Forest: How much is left, and how is the remaining forest distributed? Implications for conservation. Biological Conservation, 142(6), 1141-1153. doi: 10.1016/j.biocon.2009.02.021

Sabonaro, D. Z., \& Galbiatti, J. A. (2011). Seedling growth of Schizolobium parabyba on different substrates and irrigation levels. Rodriguésia, 62(3), 467-475. doi: 10.1590/2175-7860201162303

Scalon, S. P. Q., Mussury, R. M., Euzébio, V. L. M., Kodama, F. M., \& Kissmann, C. (2011). Estresse hídrico no metabolismo e crescimento inicial de mudas de mutambo (Guazuma ulmifolia Lam.). Ciência Florestal, 21(4), 655-662. doi: 10.5902/198050984510

Silva, J. G., Tagliaferre, C., Paula, A., Lemos, O. L., \& Rocha, F. A. (2019). Influência da lâmina de água e consumo hídrico de cinco 
essências florestais. Brazilian Journal of Irrigation and Drainage, 24(2), 303-319. doi: 10.15809/irriga.2019v24n2p303-319

Silva, L. O. C., Fonseca, A. C., Sivisaca, D. C. L., Silva, M. R., Villas Boas, R. L., \& Guerrini, I.A. (2020). Sewage sludge compost associated to frequency of irrigation for Peltophorum dubium (Sprengel) Taubert seedlings production. Floresta, 50(2), 1389-1398. doi:10.5380/rf.v50i2.64158

Siqueira, D. P., Barroso, D. G., Carvalho, G. C. M. W., Erthal, R. M., Rodrigues, M. C. C. \& Marciano, C. R. (2019). Sewage sludge treated in the substrate composition for Plathymenia reticulata Benth seedling production. Ciência Florestal, 29(2), 728-739. doi: 10.5902/1980509827297

Sobral, L. F., Barreto, M. C. V., Silva, A. J., \& Anjos, J. L. (2015). Guia prático para interpretação de resultados de análises de solo (1 $1^{\mathrm{a}} \mathrm{ed}$.). Aracaju (SE), Brasil: Embrapa Tabuleiros Costeiros.

SOS Mata Atlântica, \& Instituto Nacional de Pesquisas Espaciais [Inpe]. (2019). Atlas dos remanescentes florestais da Mata Atlântica: Período 2017-2018. Disponível em: https://www.sosma.org.br/wpcontent/uploads/2019/05/Atlas-mata-atlantica_17-18.pdf

Souchie, F. F., Junior, B. H. M., Petter, F. A., Madari, B. E., Marimon, B. S, \& Lenza, E. (2011). Carvão pirogênico como condicionante para substrato de mudas de Tachigali vulgaris L. G. Silva \& H. C. Lima. Ciência Florestal, 21(4), 811-821. doi: 10.5902/198050984526

Trigueiro, R. M., \& Guerrini, I. A. (2014). Utilização de lodo de esgoto na produção de mudas de aroeira-pimenteira. Revista Árvore, 38(4), 657-665. doi: 10.1590/S0100-67622014000400009
Wendling, I., \& Gatto, A. (2012). Substratos, adubação e irrigação na produção de mudas ( $2^{\mathrm{a}}$ ed.). Viçosa (MG), Brasil: Aprenda Fácil Editora.

Zapata, N., Guerrero, F. \& Polo, A. (2005). Evaluación de corteza de pino y residuos urbanos como componentes de substratos de cultivo. Agricultura Técnica, 65(4), 378-387. doi: 10.4067/S036528072005000400004

Manuscrito recebido em 17 de maio de 2020

Aceito em 26 de setembro de 2020

Publicado em 6 de setembro de 2021

Este documento deve ser citado como:

Pinto, V. V. F., Bueno, M. M., Antunes, L. F. S., Alonso, J. M., \& Gustavo Wyse Abaurre, G. W. (2021). Crescimento de mudas de Poincianella pluviosa (DC.) L.P.Queiroz em diferentes substratos e lâminas de irrigação. Madera y Bosques, 27(1), e2712173. doi: 10.21829/myb.2021.2712173

Madera y Bosques pelo Instituto de Ecología, A.C é distribuída sob uma Licença Internacional Creative Commons Atribuição-NãoComercial ShareAlike 4.0. 